\title{
Competencias genéricas en carreras de ingeniería
}

Adriana Isis Cerato y Monica Gallino ${ }^{1}$

\section{Resumen}

El desarrollo de la educación por competencias con base en un enfoque sistémico y complejo es una necesidad impostergable en el desarrollo de la Educación Superior en general y en el caso del ingeniero en particular para los nuevos paradigmas y desafíos del Tercer Milenio con un enfoque de Desarrollo Humano Integral

Este trabajo aborda la educación en ingeniería con un enfoque complejo e indagando a partir de hechos claves acerca de la formación por competencias, una respuesta a necesidades en educación, donde es necesario superar la inteligencia fragmentada con visiones multidimensionales e integradas. En particular se analiza la visión de los docentes del primer año de ingeniería civil sobre competencias genéricas de alumnos ingresantes.

Palabras Clave: competencias, desarrollo humano, ingeniería, ingreso. 


\section{Abstract}

The development of competency-based education under a systemic and complex focus is an urgent need for Higher Education development in general, and for engineers in particular, considering new Third Millennium paradigms and challenges with an Integral Human Development focus. This paper addresses engineering education with a complex approach and inquiring from key facts about skills training, a response to education needs, where is necessary to overcome the fragmented intelligence with multidimensional and integrated visions. In particular, it analyzes the vision of civil engineering first-year teachers about new students' generic skills.

Keywords: skills, human development, engineering, entry. 


\section{Introducción}

Este trabajo pretende ser un aporte para mejorar la enseñanza, así como, ir introduciendo a los profesores de la carrera de Ingeniería Civil directamente en la temática de los estudios en base a competencias y motivar las modalidades y metodologías de estrategias de enseñanza que facilitan su desarrollo. La programación de las actividades didácticas a proponer a los estudiantes resulta de suma importancia para que estos logren asimilación e internalización de las competencias tendientes a fortalecer en forma integral su futuro desempeño laboral profesional, tanto en lo técnico como en valores y ética profesional.

El trabajo en la Ingeniería es muy diverso en cuanto a áreas de desempeño, y poco predecible. Los ingenieros pueden trabajar en proyectos y diseños muy variados construcciones de distintos tipos y envergaduras, gestión, operaciones, desarrollo, etc. Por esta razón, clarificar competencias e implementar procesos de enseñanza que las desarrollen en forma temprana es básico para enfrentar un contexto laboral amplio y cambiante.

\section{Campo teórico y conceptual}

El concepto de competencia es diverso, según el ángulo del cual se mire o el énfasis que se le otorgue a uno u otro elemento, pero el más generalizado y aceptado es el de saber hacer en un contexto.

Por competencias se entiende el conjunto de conocimientos, habilidades, destrezas y actitudes que se integran a las características personales como capacidades, rasgos, motivos y valores y experiencias personales.

Las competencias en la educación pueden definirse como “...competencias, genéricas y especificas, entendidas como el conjunto de conocimientos, capacidades, destrezas, aptitudes y actitudes más adecuados para alcanzar unos objetivos sociales de largo recorrido. "(Suárez Arroyo, B 2005, pp. 6).

Otra forma de entender las Competencias es movilizando el conjunto de saberes: el saber (disponer de un conjunto de conocimientos para realizar una tarea), el saber hacer (poseer habilidades para aplicar y utilizar los conocimientos), y el saber estar o saber ser (referido a las actitudes y valores) (Delors, 1996).

Edgar Morín (1999) enuncia los siguientes aspectos:

- Tener en cuenta las limitaciones del conocimiento humano

- Adquirir un conocimiento global y contextualizado de los temas

- Conocer las características de la condición humana

- Saber vivir en un mundo globalizado, interrelacionado, cambiante. 
- Aprender a afrontar las incertidumbres y que la solución de unos problemas genera otros.

- Ser comprensivo ante los demás seres humanos, en este mundo que conlleva muchos más contactos con personas de diversa condición.

- Disponer de una formación ética, que deberá obtenerse mediante un ejercicio constante de reflexión y práctica democrática.

- Pensamiento Complejo, el estudio de un fenómeno se puede hacer desde la dependencia de dos perspectivas: holística y reduccionista. La primera, se refiere a un estudio desde el todo o todo múltiple; y la segunda, a un estudio desde las partes

Tobon (2004) define a las competencias, como procesos complejos de desempeño con idoneidad en determinados contextos, integrando diferentes saberes (saber ser, saber hacer, saber conocer y saber convivir).

Todo esto para realizar actividades y/o resolver problemas con sentido de reto, motivación, flexibilidad, creatividad, comprensión y emprendimiento, dentro de una perspectiva de procesamiento metacognitivo, mejoramiento continuo y compromiso ético.

Siendo la meta contribuir al desarrollo personal, la construcción y afianzamiento del tejido social, la búsqueda continua del desarrollo económico-empresarial sostenible, y el cuidado y protección del ambiente y de las especies vivas.

Las competencias desde la perspectiva compleja se posicionan en la categoría general del desarrollo humano consistiendo la misma en el conjunto de características biopsicosociales propias de cada persona en la búsqueda del bienestar y la autorrealización de acuerdo a condiciones personales y del contexto en que se encuentra inserto.

Según Suarez y Arroyo(2005) "Todo parece indicar que en una visión moderna de las profesiones y de la educación, la formación en competencias en su versión más trascendente a lo largo de la vida, la experiencia en el trabajo y la madurez personal y profesional deberían ser los factores que faciliten a los titulados de hoy crecer y progresar en unas competencias profesionales cambiantes día a día y cada vez más complejas; esta es una cuestión fundamental para construir una sociedad de ciudadanos más justa dónde el bienestar sea un elemento clave en el desarrollo de la vida cotidiana" (pp.4).

Entre los distintos conceptos y lo planteado por Morín se encuentran dos premisas de políticas educativas con distintas definiciones: competencias para el Desarrollo de Capital Humano o competencias para el Desarrollo Humano.

El Desarrollo de Capital Humano relaciona a las competencias con la capacidad de las personas en el desempeño de las actividades o funciones de su puesto de trabajo.

En cambio, si el objetivo es el Desarrollo Humano se necesitan competencias que fortalezcan en forma integral a las personas y su inserción en la sociedad.

Por ello, es vital la vigencia o la creación, si no las hubiere, de políticas de Estado que tengan como meta el desarrollo humano.

Dado todo lo mencionado previamente, la incorporación del desarrollo de 
competencias en la enseñanza de la ingeniería requiere un trabajo previo de análisis y discusión. Esto implica, por un lado, un trabajo de puesta en común de los cambios u objetivos buscados, con los docentes y, por otro, un programa institucional de formación y capacitación para todos los involucrados. Un sistema de apoyo y seguimiento que debe operar previo y durante la implementación de forma de asegurar una trasformación real en el cumplimiento de los objetivos buscados.

De lo contrario se corre el riesgo de caer en una visión simplista que termine en el mejor de los casos, en una tabla que relacione contenidos con competencias y una suma de nuevas actividades a las tradicionalmente realizadas, sin lograr el fin buscado.

Según Cesar Coll (2007): "tal vez el riesgo principal del enfoque basado en competencias sea similar al que han tenido que afrontar en el pasado otros enfoques, con éxito casi siempre más bien escaso o moderado: el de presentarse y ser presentado como una solución a los males, problemas e incertidumbres que aquejan la educación escolar en la actualidad. Las aportaciones de los enfoques basados en competencias son muy valiosas, pero definitivamente tampoco son un remedio milagroso" (pp.39).

La formación basada en competencias requiere asumir una nueva racionalidad que trascienda la parcelación y la fragmentación, con el fin de abordar la realidad en su multidimensionalidad (Morín, 2000): "La inteligencia parcelada, compartimentada, mecanicista, disyuntiva, reduccionista rompe lo complejo del mundo en fragmentos separados, fracciona los problemas, separa lo que está unido, unidimensionaliza lo multidimensional. Es una inteligencia miope que termina normalmente por enceguecerse.... incapaz de proyectar el contexto y el complejo planetario, la inteligencia ciega se vuelve inconsciente e irresponsable” (pp.34).

Desarrollar el pensamiento complejo, implica que el estudio de un fenómeno, se puede hacer desde dos perspectivas: holística y reduccionista. La primera, se refiere a un estudio desde el todo múltiple, y la segunda a un estudio desde las partes.

Los desafíos en la educación son los dirigidos a aplicar modos y metodologías de estrategias de enseñanza, que tiendan a una educación transdisciplinaria, desarrollo de habilidades metacognitivas, más que acumulaciones de conocimientos de hechos. Saber qué hacer con lo que se sabe, formación de pensamiento esencialmente científico, apertura al pensamiento intuitivo, creatividad, formación de valores, dar respuesta a las necesidades de diversos agentes sociales, etc. En síntesis desarrollar competencias para el Desarrollo Humano Integral.

\section{Visión de profesores de ingeniería civil de primer año respecto a competencias genéricas de alumnos ingresantes.}

En el presente trabajo se presentan en forma sintética algunas de los análisis y conclusiones a las que se arribaron en una investigación de mayor alcance que 
involucro una metodología de la investigación con un enfoque cuali-cuantitativo que permitió realizar un estudio exploratorio correlacional de competencias genéricas y estrategias de enseñanza aplicadas a la enseñanza de la ingeniería civil en la UNC por parte del conjunto de profesores de las materias del primer año. Con respecto a las competencias genéricas se utilizaron las acordadas en el proyecto Alfa Tunning (2007).

El proyecto Alfa Tuning (2007) define competencia como las "capacidades que todo ser humano necesita para resolver, de manera eficaz y autónoma, las situaciones de vida. Se fundamentan en un saber profundo, no sólo saber qué y saber cómo, sino saber ser persona en un mundo complejo cambiante y competitivo. "(pp.35).

Establece que sobre las competencias genéricas, se busca identificar aquellos atributos compartidos que pudieran generarse en cualquier titulación y que son considerados importantes por la sociedad además de ser comunes a todas o casi todas las titulaciones. Las competencias específicas son las que se relacionan con cada área temática, y tienen una gran importancia para cualquier titulación al estar específicamente relacionadas con un conocimiento concreto.

Las competencias genéricas del estudio de referencia son:

1. Capacidad de abstracción, análisis y síntesis

2. Capacidad de aplicar los conocimientos en la práctica

3. Capacidad para organizar y planificar el tiempo

4. Conocimientos sobre el área de estudio y la profesión

5. Responsabilidad social y compromiso ciudadano

6. Capacidad de comunicación oral y escrita

7. Capacidad de comunicación en un segundo idioma

8. Habilidades en el uso de las tecnologías de la información y de la comunicación

9. Capacidad de investigación

10. Capacidad de aprender y actualizarse permanente

11. Habilidades para buscar, procesar y analizar información procedente de fuentes diversas

12. Capacidad crítica y autocrítica

13. Capacidad para actuar en nuevas situaciones

14. Capacidad creativa

15. Capacidad para identificar, plantear y resolver problemas

16. Capacidad para tomar decisiones

17. Capacidad de trabajo en equipo

18. Habilidades interpersonales

19. Capacidad de motivar y conducir hacia metas comunes

20. Compromiso con la preservación del medio ambiente

21. Compromiso con su medio socio-cultural 
22. Valoración y respeto por la diversidad y multiculturalidad

23. Habilidad para trabajar en contextos internacionales

24. Habilidad para trabajar en forma autónoma

25. Capacidad para formular y gestionar proyectos

26. Compromiso ético

27. Compromiso con la calidad

\section{Competencias genéricas caso alumno ingresante}

Los alumnos de primer año en general provienen directamente del nivel medio de educación, con un inicio en sus estudios universitarios con el ciclo de nivelación de solo dos meses, por lo cual se puede inferir que las capacidades con que afrontan sus estudios universitarios provienen de los procesos formativos desarrollados en niveles educativos anteriores.

En ese aspecto, es necesario determinar para el análisis cual es la percepción de los profesores de primer año en cuanto al desafío para iniciar la formación universitaria en ingeniería en función de clarificar el perfil del nuevo estudiante universitario de acuerdo a las competencias genéricas con las que ingresa.

Sobre las 27 competencias ya enumeradas, se interrogó a los profesores sobre el grado de desarrollo de las mismas de los alumnos de primer año. Se consideraron cuatro categorías desde un mínimo de ningún desarrollo ( $1^{\mathrm{a}}$ categoría) con intervalos de baja ( $2^{\mathrm{a}}$ categoría), bastante ( $3^{\mathrm{a}}$ categoría) a mucho ( $4^{\mathrm{a}}$ categoría).

En este aspecto todas se encuentran entre el rango 1 y 2, salvo la competencia de habilidades interpersonales que supera aunque en muy poca medida el valor de poco desarrollo (Figura 1).

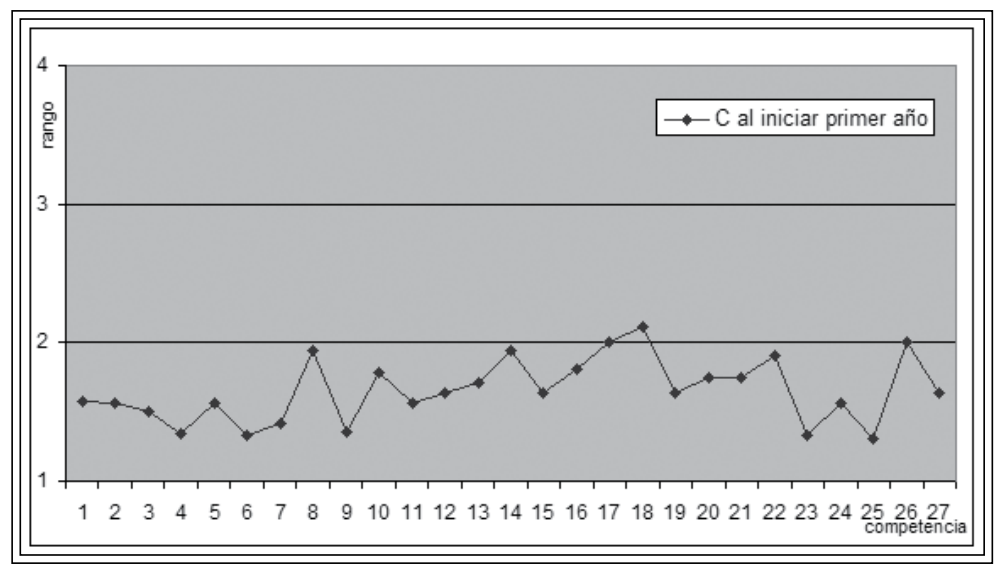

Figura 1 Curva de desarrollo de competencias genéricas en alumnos ingresantes Fuente: Elaboración Propia | Escala 1 nada 2 poco 3 bastante 4 mucho 
A partir del análisis precedente se puede concluir que en lo que respecta a competencias genéricas, según la percepción de los profesores universitarios de primer año, los aportes de niveles educativos anteriores son muy bajos, lo que dificulta los procesos de formación en la universidad y un gran esfuerzo de los cursos al inicio de la carrera.

\section{Las competencias genéricas en el ingreso y el CONFEDI}

El Consejo Federal de Decanos de Ingeniería (CONFEDI) de la República Argentina y otras instituciones afines tales como: Asociación Universitaria de Educación Superior Universitaria (AUDEAS), Consejo Nacional de Decanos de Veterinaria (CONADEV), Consejo Universitario de Ciencias Exactas y Naturales( CUCEN), Ente coordinador de Unidades Académicas de Farmacia y Bioquímica (ECUAFyB), Foro de Decanos de las Facultades de Química (FODEQUI) y Red de Universidades con carreras en Informática (RED UNCI), desarrollan una propuesta sobre las competencias necesarias de acceso de un estudiante de nivel medio que desea continuar estudios superiores en el área.

En dicho documento (CONFEDI 2009) se expresa:

"Las características de la Educación Superior requieren que quien inicia una carrera universitaria deba poseer el dominio de una serie de competencias básicas entre las cuales cumple un papel muy importante el manejo de las formas más complejas del lenguaje. Asimismo el aprendizaje constituye un proceso complejo que se compone de competencias diferentes que convergen en el resultado final formativo. Tal como lo plantean los documentos que organizan y definen las competencias para los egresados de la escuela secundaria, las capacidades creativas y de resolución de problemas, asi como el pensamiento complejo, están dados por operaciones mentales mediadas y transmitidas culturalmente por el lenguaje en sus diferentes concepciones.

La formación de los estudiantes en el nivel medio, debe desarrollar competencias generales como: creatividad, interés por aprender, pensamiento crítico (capacidad de pensar con juicio propio) habilidad comunicacional, capacidad para resolver situaciones problemáticas, tomar decisiones, adaptarse a los cambios y trabajar en equipo, poseer pensamiento lógico y formal.

Estas competencias deben ser desarrolladas en la escuela secundaria y durante la instancia universitaria continuar con su desarrollo y consolidación" (pp.1).

En este documento también se definen las competencias de Acceso que se visualizan en Figura $\mathrm{N}^{\mathrm{o}} 2$.

Se dividen en tres grupos:

Competencias Básicas están referidas a los conocimientos, procedimientos, destrezas y actitudes fundamentales para el desarrollo de otros aprendizajes 
Competencias Transversales están referidas a la capacidad para regular sus propios aprendizajes, aprender solos y en grupo, y resolver las dificultades a que se ven enfrentados durante el transcurso del proceso de aprendizaje

Competencias Específicas Referidas a un conjunto de capacidades relacionadas entre sí, que permiten desempeños satisfactorios en el estudio de las carreras

\begin{tabular}{|l|l|l|}
\hline \multicolumn{1}{|c|}{ Competencias Básicas } & $\begin{array}{l}\text { Competencias } \\
\text { Transversales }\end{array}$ & Competencias Específicas \\
\hline $\begin{array}{l}\text { Aluden a capacidades com- } \\
\text { plejas y generales necesa- } \\
\text { rias para cualquier tipo de } \\
\text { actividad intelectual. }\end{array}$ & $\begin{array}{l}\text { Aluden a capacidades } \\
\text { claves para los estu- } \\
\text { dios superiores. }\end{array}$ & $\begin{array}{l}\text { Remiten a un conjunto de capaci- } \\
\text { dades relacionadas entre si, que } \\
\text { permiten desempeños satisfactorios } \\
\text { en el estudio de las carreras. }\end{array}$ \\
\hline
\end{tabular}

\begin{tabular}{|c|c|c|}
\hline $\begin{array}{l}\text { 1. Compresión lectora } \\
\text { 2. Producción de textos } \\
\text { 3. Resolución de } \\
\text { problemas }\end{array}$ & $\begin{array}{l}\text { 1. Autonomía en el } \\
\text { aprendizaje } \\
\text { 2. Destrezas cogniti- } \\
\text { vas generales }\end{array}$ & $\begin{array}{l}\text { 1. Análsis de una función o un } \\
\text { fenómeno fíco y/o químico sencillo } \\
\text { a partir de su representación gráfica } \\
\text { y/o a partir de sus ecuaciones mate- } \\
\text { máticas. } \\
\text { 2. Reconocimiento y utilización de } \\
\text { conceptos en matemática, física, } \\
\text { química y biología } \\
\text { 3. Reconocimiento y análisis de } \\
\text { propiedades físicas y/o químicas de } \\
\text { la materia en ejemplos cotidianos. } \\
\text { 4. Tranferencia del conocimiento } \\
\text { científico de física, química, matemá- } \\
\text { tica y biología a situaciones proble- } \\
\text { máticas variadas } \\
\text { 5. Utilización de la comptadora } \\
\text { aplicando lógica procedimental en } \\
\text { la utilización del Sistema Operativo y } \\
\text { diversas aplicaciones como: Proce- } \\
\text { sador de textos, Internet y Correo } \\
\text { Electrónico }\end{array}$ \\
\hline
\end{tabular}

Figura 2 Competencias de acceso a estudios Universitarios | Fuente: Documento sobre Competencias requeridas para el Ingreso a los Estudios Universitarios CONFEDI (2009)

En el citado documento se establece que el nivel esperado de las competencias de acceso definidas a estudios superiores sea entre intermedio y alto.

A continuación se indican de acuerdo a grupos y subgrupos cuales son los niveles esperados de desarrollo de competencias de acceso en un ingresante:

\section{Competencias básicas}

1. Comprensión lectora: Intermedio

2. Producción de textos: Intermedio

3. Resolución de problemas: Intermedio 


\section{II- Competencias transversales}

1. Autonomía en el aprendizaje. : Intermedio Alto

2. Destrezas cognitivas generales: Intermedio

3. Relaciones interpersonales: Intermedio

\section{Competencias específicas: Alto}

En base a los resultados de los niveles de desarrollo de competencias genéricas de los estudiantes al ingresar, según la percepción de los profesores de primer año, se realizó una correlación con las Competencias de acceso establecidas por CONFEDI, en base a la correspondencia las competencias genéricas enumeradas en el punto 3, con el fin de extraer conclusiones respecto a la situación de un novel estudiante al acceder a estudios de Educación Superior. Se aclara que respecto al grupo de competencias específicas la correlación está limitada solo a competencias genéricas, no a conocimiento sobre contenidos específicos (matemática, física, etc.) que no estaban dentro del alcance de la investigación.

El nivel de desarrollo un ingresante, de acuerdo a la percepción de los profesores de primer año, de las Competencias de Acceso se ubica en las escalas más bajas entre niveles por debajo de poco conocimiento.

Si realizamos una correlación entre los niveles necesarios o deseados de las Competencias de Acceso establecidas por CONFEDI se llega a que están muy lejos de las efectivas de un estudiante que proviene del nivel medio de educación (Figura 3).

El salto cualitativo entre lo real (según la percepción de los profesores de primer año) y lo deseado es muy alto, lo que trae como consecuencias un fuerte trabajo de los profesores de primer año para que los alumnos superen las falencias de niveles educativos anteriores, así como produce desgranamiento y deserción de estudiantes por falta de capacidades mínimas para el desarrollo de estudios superiores.

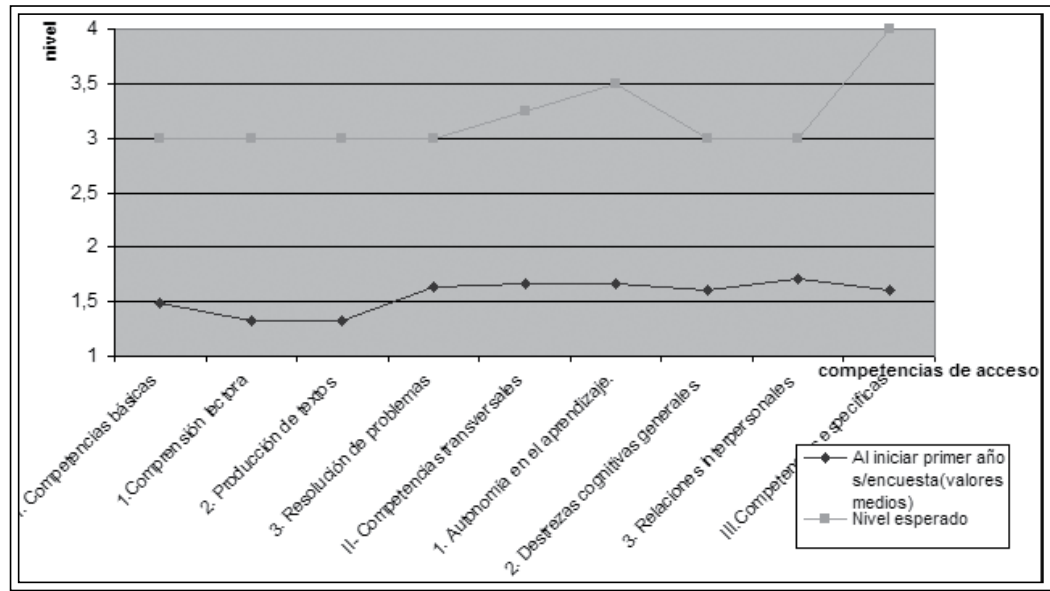

Figura 3 Correlación de niveles de Competencias de acceso deseadas y reales Fuente: Elaboración Propia | Escala 1 nada 2 poco 3 bastante 4 mucho 


\section{Conclusiones}

La distancia entre el deseo y la realidad se hacen patentes en la necesidad de posibilitar el desarrollo de habilidades básicas y específicas, que en primer año se presenta como una ardua tarea. Es allí donde se observa además un mayor requerimiento de estrategias de enseñanza que favorezcan buenos aprendizajes desde la generación de procesos cognitivos y saberes prácticos, en donde no basta revalorizar el contenido sino el conocimiento didáctico del contenido.

De las visiones del desarrollo de competencias, desde el punto de vista de la percepción de los profesores de primer año, que ya trae el ingresante de otros niveles educativos se desprende que el salto cuantitativo y cualitativo que se debe dar en Educación Superior y específicamente en el caso de un futuro ingeniero civil es de gran magnitud.

Si bien en este artículo se presenta solo una parte de la investigación de mucho mayor alcance para evaluar el marco conceptual se trata de expresar los puntos de partida para desarrollar pensamiento complejo en estudios universitarios.

Por ello es necesario profundizar políticas de articulación del nivel medio con Educación Superior .Además de impulsar la sensibilización del cuerpo docente de perfeccionar cada vez más sus estudiantes para que egresen con la calidad requerida-, de las necesidades y la constante competencia que el mundo de hoy exige.

El desarrollo de la educación por competencias, con base en un enfoque sistémico y complejo es necesaria como aporte en el desarrollo de la Educación Superior en general y en el caso del ingeniero en particular, para enfrentar nuevos paradigmas y desafíos 


\section{Bibliografía}

Autor (2012) "Consejo Federal de Decanos de Ingeniería (2009) Documento sobre Competencias requeridas para el Ingreso a los Estudios Universitarios Disponible en URL http://confedi.org.ar/documentos/competencias-de-ingreso [consulta 3 de noviembre de 2011]

Coll , C (2007) Las competencias en la educación escolar: algo más que una moda y mucho menos que un remedio Revista Aula de Innovación Educativa. Núm. 161Editrial Grao pp39

Delors, J (1996) “La educación encierra un tesoro” Editorial Santillana Ediciones Unesco

Morín, E-(1999) Los siete saberes necesarios para la educación del futuro, Organización de las Naciones Unidas para la Educación, la Ciencia y la Cultura. Ediciones UNESCO Francia

Morín, E. (2000). Los siete saberes necesarios para la educación del futuro. Bogotá: Ministerio de Educación Nacional'.

Proyecto Alfa Tunig (2007) Informe Final Reflexiones y Perspectivas de la Educación Superior en América Latina, Editor Universidad de Deusto España.

Suarez y Arroyo, B (2005) La formación en competencias: un desafio para la educación superior del futuro, Universidad Politécnica de Cataluña Barcelona Tobón, S (2004) Formación basada en competencias. Pensamiento complejo, diseño curricular y didáctica. Colombia. Esfera Editores. 